\title{
Conservatism of host specificity in parasites
}

\author{
David Mouillot, Boris R. Krasnov, Georgy I. Shenbrot, Kevin J. Gaston and Robert Poulin
}

Mouillot, D., Krasnov, B. R., Shenbrot, G. I., Gaston, K. J. and Poulin, R. 2006. Conservatism of host specificity in parasites. - Ecography 29: 596-602.

\begin{abstract}
As information becomes available for many groups of organisms a general pattern of phylogenetic conservatism in ecological characters or morphological traits is now widely recognized. Conversely, conservatism of external ecological attributes throughout a lineage is still a contentious theme in ecology. Moreover, the studies exploring this topic have focused on free-living organisms, and have ignored parasites. The main external ecological attribute of parasite species is certainly their host specificity, which is a key determinant of both their range size and local abundance. We address the subject of conservatism and predictability of host specificity using 2 large databases concerning, respectively, ectoparasites and endoparasites. We found a significant positive relationship between the numbers of host species infested by flea sister species. Moreover, this result was consistent whether we used sympatric or allopatric flea species, suggesting no influence of the mode of speciation on this conservatism of specificity. Additionally, our results showed that congeneric helminth species have more similar host taxonomic diversities than expected by chance, although this conservatism is due mostly to trematodes. Whilst there is evidence of conservatism, the moderate levels preclude robust prediction of host specificity for one species based on that of closely related species.
\end{abstract}

D. Mouillot (mouillot@univ-montp2.fr) and K. J. Gaston, Biodiversity and Macroecology Group, Dept of Animal and Plant Sciences, Univ. of Sheffield, Sheffield S10 2TN, UK (present address of D. M.: UMR CNRS-UMII 5119 Ecosystemes Lagunaires, Univ. of Montpellier II, CC093, FR-34095 Montpellier Cedex 5, France). - B. R. Krasnov and G. I. Shenbrot, Ramon Science Center and Mitrani Dept of Desert Ecology, Jacob Blaustein Inst. for Desert Research, Ben-Gurion Univ. of the Negev, P.O. Box 194, Mizpe Ramon IL-80600, Israel. - R. Poulin, Dept of Zoology, Univ. of Otago, P.O. Box 56, Dunedin, New Zealand.

Theory predicts niche conservatism among species over evolutionary time scales; i.e. closely related species should tend to share common ecological attributes inherited from a common ancestor (Peterson et al. 1999). This conservatism may be due to active, stabilizing selection or to an evolutionary reduction in the potential range of ecological characters caused by the fixation of ancestral traits (i.e. developmental constraints). Whereas conservatism of morphological traits such as body size has been widely reported (Desdevises et al. 2003), the conservatism of external attributes such as migratory behaviour (Bohning-Gaese and Oberrath
2003), dietary niche breadth (Brandle et al. 2002), and geographic range size (Webb and Gaston 2003) are still under scrutiny because accidents of history and geography certainly impact such species attributes (Brown 1995, Gaston and Blackburn 2000). The mode of speciation may also affect the conservatism of external attributes because sister species sharing morphological traits are perhaps more likely also to share external attributes when they live in sympatry than when they live in allopatry because they evolve in a similar environment. Thus new empirical investigations including closely related species both of sympatric and allopatric

Accepted 7 March 2006 
origin are required to disentangle the influence of biological conservatism and environment on species external attributes.

Parasite species offer interesting opportunities to assess the conservatism of external ecological attributes with observed datasets including both sympatric and allopatric species, because sympatric speciation may be more common in parasitic than in free-living animals (Théron and Combes 1995, de Meeûs et al. 1998, McCoy 2003). Host specificity has been recognized as a "true" parasite character evolving under constraints such as host characteristics and it increases as parasites infest 1) a low number and 2) a narrow phylogenetic range of host species (Poulin and Mouillot 2003), the latter providing an evolutionary perspective on specificity. Host specificity is a key determinant of many basic properties of parasitic organisms, such as their ability to switch to new host species (Holt et al. 2003), their probability of extinction (Holt et al. 2003, Koh et al. 2004), their range size (Krasnov et al. 2005), and their local abundance (Krasnov et al. 2004a, b); thus, understanding the evolutionary heritability of host specificity is crucial to many applied issues. For example, the level of host specificity shown by parasites has profound implications for their population dynamics, for epidemiology, and the likelihood that they can switch to new host species, including humans (Bush and Kennedy 1994, Poulin 1998, Morand and Guégan 2000).

The aim of science is to understand systems to the extent that predictions can be made about their functioning; our ultimate objective is thus to predict the specificity of one species from that of closely related species. This goal is of great relevance to parasite macroecology, but also to more applied branches of parasitology. Host specificity measures the range of tolerable conditions or environments for a parasite, and its evolution matters to an understanding of parasites and our ability to control them. Rohde (1979) pointed out early on that species of the same genus usually, though by no means invariably, have some similarity in their hosts and in the range of their hosts. Nevertheless, despite some attempts suggesting either a limited role of phylogeny on parasitoid host ranges (Stireman and Singer 2003) or similarities in host specificity within parasite taxonomic groups (Sasal et al. 1998), conservatism through evolutionary lineages of this fundamental ecological attribute of parasites has never been investigated per se on large datasets. Previous results with fleas suggest that host specificity is to a large extent phylogenetically constrained, while still strongly influenced by local environmental conditions (Krasnov et al. 2004a, b). We thus predict conservatism of host specificity but mainly for sympatric species more likely susceptible to share host species and similar environmental conditions. The aim of this study is to address the questions: 1) are the host specificities of closely related species more similar to one another than expected from randomly associated pairs of species? 2) Are the host specificities of parasite species predictable? These questions are investigated using 2 datasets, one on ectoparasites and one on endoparasites.

\section{Material and methods}

\section{Flea data}

We collected data from published regional surveys that reported flea distribution on small mammals in 35 regions across the globe, over a 50-yr period (1955-2003), in which 399367 individual small mammals (Didelphimorphia, Insectivora, Lagomorpha, and Rodentia) of 222 species were examined (see Krasnov et al. 2004a, b for details). These sources provided data on the number of individuals of each flea species found on a given number of individuals of each particular host species, except in the Barguzin Depression region, for which data on the average abundance of a flea species on a host species were provided instead (Vershinina et al. 1967). Single findings of a flea species on a host species or in a region were considered accidental and were not included in the analysis. In total, we used data on 297 flea species and we extracted 68 pairs of sister species from a phylogeny based on the taxonomy of the entire flea fauna of the world proposed in Hopkins and Rothschild (1953, 1956, 1962, 1966, 1971), Traub et al. (1983), and Smit (1987). These 68 pairs of sister species included both sympatric sister species that occurred in the same region, and allopatric sister species that occurred in different geographical regions. Sympatry or allopatry of fleas was established based on the global occurrences of the respective sister pairs (see Krasnov et al. 2004a for details).

\section{Helminth data}

The second dataset comes from a survey of helminth bird parasites in Azerbaijan, in which $>6000$ individual birds from 158 different species were examined for intestinal helminths (Vaidova 1978). Data were available at the species level for trematodes, cestodes, and nematodes. For the bird species included, the mean \pm SE number of individuals examined per species was $46 \pm 12$. We excluded parasite species for which the mean number of individuals examined per bird species was $<15$. Pairs of parasite species were made up of congeneric species in the database. When there were $>2$ species in a genus, we included all possible pairs of species from that genus. Although this meant that some species were used in more than one pair, the permutation 
method used for the statistical analyses (see below) removed any bias that may be associated with the nonindependence of data points. In total we thus obtained 580 pairs of congeneric helminth species.

\section{Specificity estimations}

The classical measure of host specificity is the number of host species on which the parasite species was found. For the helminth data the sample size (number of host individuals examined) had no influence on this measure of specificity (Poulin and Mouillot 2004). For the fleas, however, the number of host individuals examined was not homogeneous among regions and could bias the results. Thus, the number of mammalian species on which a flea species was found (specificity) was corrected for sampling effort and we considered the residuals of the regression in the following analyses.

In addition, rather than taking only the number of host species used by a parasite as a measure of its host specificity, we also applied a measure of host specificity that takes into account the taxonomic or phylogenetic affinities of the various host species (Poulin and Mouillot 2003). When these host species are placed within a taxonomic hierarchy, the average taxonomic distinctness is simply the mean number of steps up the hierarchy that must be taken to reach a taxon common to 2 host species, computed across all possible pairs of host species. The greater the taxonomic distinctness between host species $\left(\mathrm{S}_{\mathrm{TD}}\right)$, the higher the number of steps needed, and the higher the value of the index; thus it is inversely proportional to host specificity. A high index value means that on average the hosts of a parasite species are not closely related. However, the index cannot be computed for parasites exploiting a single host species.

Using the taxonomic classification of Wilson and Reeder (1993), all mammal species infested by fleas were placed into a taxonomic structure with 5 hierarchical levels above species (i.e. genus, subfamily, family, order, and class). Using the taxonomic classification of Sibley and Monroe (1990), which is based on phylogenetic relationships among bird taxa (Sibley and Ahlquist 1991), all bird species infested by helminths are in the infraclass Neoaves, and there are 12 taxonomic levels above species (i.e. genus, tribe, subfamily, family, superfamily, parvorder, infraorder, suborder, order, superorder, parvclass, and infraclass). Different taxonomic levels were used for the 2 databases because the taxonomic schemes used for birds and mammals have a different structure, with that for birds including more levels of subdivisions adding more information.

\section{Statistical analysis}

To test the conservatism of attributes such as host specificity, we used the coefficients of correlation between values for pairs of sister species for fleas and pairs of congeneric species for helminths. In each pair of closely related species there is obviously no reason to have a dependent one and an independent explanatory one and an explained one and thus no reason to have one on the abscissa and the other one on the ordinate. Nevertheless the choice of the order of species in the pair influences the value of the correlation coefficient. To overcome this problem, we used a mean correlation coefficient for each set of closely related species being compared. We randomly swapped the order of the closely related species in each pair to obtain 1000 coefficients of correlation, with the mean of the series providing an unbiased estimate of the true correlation. Thereafter we tested for the departure of this observed correlation coefficient from the null hypothesis stipulating that the observed coefficient between closely related species was not different from that calculated among species randomly associated in pairs. We implemented a Monte-Carlo procedure randomly creating species pairs from which we estimated 9999 randomized coefficients of correlation. The probability of rejecting the null hypothesis when true was calculated by comparing the distribution of coefficients of correlation obtained under the null model with the observed correlation coefficient (Manly 1998). The result is assumed to be significant (rejection of the null hypothesis) when the p-value is $<5 \%$.

\section{Results}

From the regional databases we extracted 68 pairs of flea sister species and 580 pairs of congeneric helminth species. Among the 68 pairs of flea species were 39 pairs of allopatric species and 29 pairs of sympatric species. The 580 pairs of congeneric helminth species consisted of 105 pairs of cestode, 134 of nematode, and 341 pairs of trematode species. Of the 580 pairs of congeneric helminths used in the analysis, 78 pairs included species that parasitized only one host species each; in all other pairs at least one of the 2 species in a pair parasitized $>1$ host.

For the flea species, host specificity, expressed in terms of residuals of number of host species, was significantly conserved among sister species; observed pairs of sister flea species show a significantly higher correlation coefficient than randomly associated pairs of species from the pool of fleas (Table 1). This result supports the existence of evolutionary conservatism for this external attribute for both sympatric and allopatric species (tested separately for the 2 groups). Conversely, the 
Table 1. Correlation coefficients between host specificities of closely related species of fleas and helminth species, and tests of their departure from the null hypothesis that the observed coefficient between closely related species is not different from that calculated among random species pairs. Mean host specificity and standard deviation (SD) is provided for each group of parasites. This specificity was expressed in terms of host number and host taxonomic diversity $\mathrm{S}_{\mathrm{TD}}$. The p-values were obtained using 9999 permutations and the significant results are in bold.

\begin{tabular}{|c|c|c|c|c|c|c|c|c|}
\hline & \multicolumn{4}{|c|}{ Host number } & \multicolumn{4}{|c|}{$\mathrm{S}_{\mathrm{TD}}$} \\
\hline & $\mathrm{r}$ & $\mathrm{p}$ & mean $(\mathrm{SD})$ & $\mathrm{n}$ & $\mathrm{r}$ & $\mathrm{p}$ & mean $(\mathrm{SD})$ & $\mathrm{n}$ \\
\hline \multicolumn{9}{|l|}{ Fleas } \\
\hline All & 0.43 & 0.0002 & $3.52(0.2)$ & 68 & 0.1 & 0.2593 & $2.00(0.11)$ & 49 \\
\hline Allopatric & 0.37 & 0.0074 & $3.00(0.23)$ & 39 & -0.02 & 0.4603 & $1.88(0.16)$ & 24 \\
\hline Sympatric & 0.46 & 0.0098 & $4.21(0.32)$ & 29 & 0.26 & 0.1007 & $2.14(0.15)$ & 25 \\
\hline \multicolumn{9}{|l|}{ Helminth } \\
\hline All & -0.02 & 0.3623 & $3.44(0.1)$ & 580 & 0.23 & 0.0005 & $2.99(0.09)$ & 239 \\
\hline Cestode & 0.08 & 0.2058 & $2.70(0.15)$ & 105 & 0.13 & 0.1935 & $3.58(0.28)$ & 47 \\
\hline Nematode & -0.01 & 0.483 & $3.72(0.21)$ & 134 & 0.04 & 0.3807 & $2.94(0.18)$ & 60 \\
\hline Trematode & -0.04 & 0.2129 & $3.56(0.15)$ & 341 & 0.29 & 0.0004 & $2.84(0.12)$ & 132 \\
\hline
\end{tabular}

taxonomic diversity of host species was not significantly conserved for flea species (Table 1).

In contrast, for congeneric helminth species the taxonomic diversity of host species was significantly conserved while the number of host species was not (Table 1). The conservatism observed for helminth parasite species was not similar for the different taxonomic groups, however. The result obtained for the entire assemblage was due mainly to conservatism observed for trematodes. For the cestodes and nematodes conservatism of host specificity was not apparent, regardless of the specificity metric used.

Can we predict the host specificity of a species from the specificity of its close relatives or that of its ancestor? To this end, we focused on the linear relations between the host specificities of closely related species in cases where conservatism was demonstrated; the coefficient of determination $\left(\mathrm{R}^{2}\right)$ obtained provided insight into the phylogenetic component of host specificity. For sister flea species, the explained variation in number of host species varied from $14.4 \%$ for allopatric pairs of species to $21.2 \%$ for sympatric pairs (Fig. 1). For congeneric helminth species the explained variation in host taxonomic diversity varied from $0.1 \%$ for nematodes to $8.4 \%$ for trematodes, and was only $5.3 \%$ for the whole dataset (Fig. 2). Thus, although host specificity in parasites shows significant conservatism, this conserved element is not strong enough to allow any accurate prediction.

\section{Discussion}

Our study demonstrates that specificity in terms of number of host species is conserved for flea species and that specificity in terms of taxonomic diversity of hosts is conserved for congeneric helminth species. This conservatism is highly heterogeneous in helminth parasites where it is due mostly to the trematodes, but is not apparent for cestodes and nematodes. Conservatism of host specificity is not affected by the probable mode of speciation as suggested from the present geographical distributions of the parasites. One might suspect that sympatric sister flea species are more likely to share biological traits, functional attributes, and host species used as habitats. Of course, caution is needed when making inferences based on contemporary sympatry. Sympatric sister species can be secondarily sympatric: they could have become sympatric after an initial allopatric speciation event (Via 2001). Still, although living in sympatry does not necessarily result from sympatric speciation, it is likely that sympatric speciation is a major reason why fleas now live in sympatry. We observed that the taxonomic diversity of hosts is not significantly more similar between sympatric sister flea species than between allopatric species (Table 1). Thus, mode of speciation seems to be secondary in its influence on the observed conservatism of host specificity in fleas; the main factor is phylogeny, as predicted by Krasnov et al. (2004b).

The level of conservatism or heritability of host specificity in helminths depends on the taxa involved and on whether specificity refers simply to the number of host species or the taxonomic diversity of host species. Of all the differences between these taxa, one in particular, mode of transmission, may be important in this difference. Helminths are transmitted to their bird hosts via predation on intermediate hosts while fleas possess a direct life cycle. Thus, from the helminth perspective, for a new host to be suitable, it must not only have the right diet, but the "living conditions" inside must also be acceptable for the parasites; e.g. the immune response must be similar to that in the original host. Perhaps this does not impose limits on the number of potential host species, while at the same time constraining the taxonomic relatedness of these hosts. Other explanations are possible, although their causal importance cannot be tested. The explanation for the 


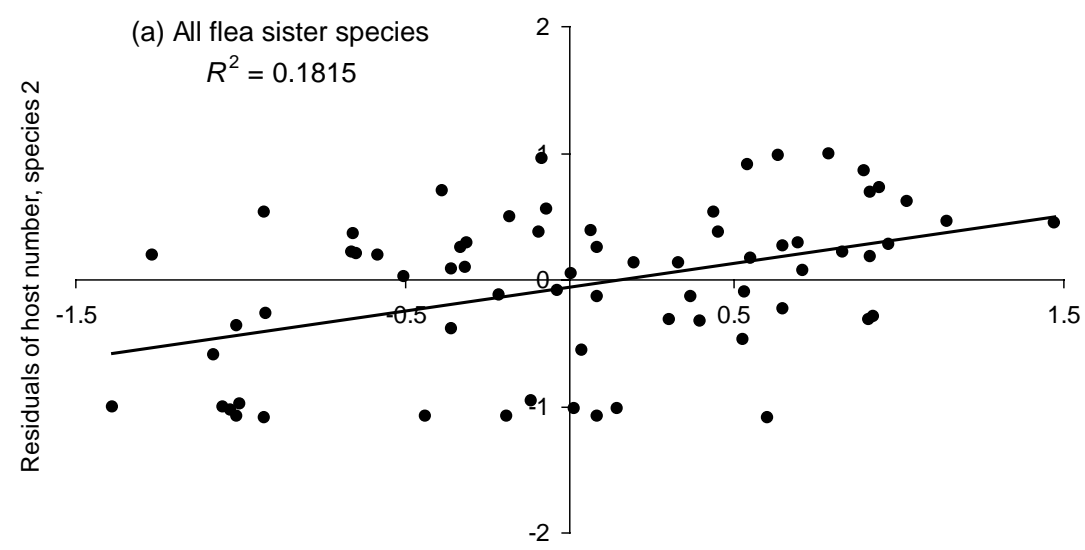

Residuals of host number, species 1

(b) Allopatric flea sister species

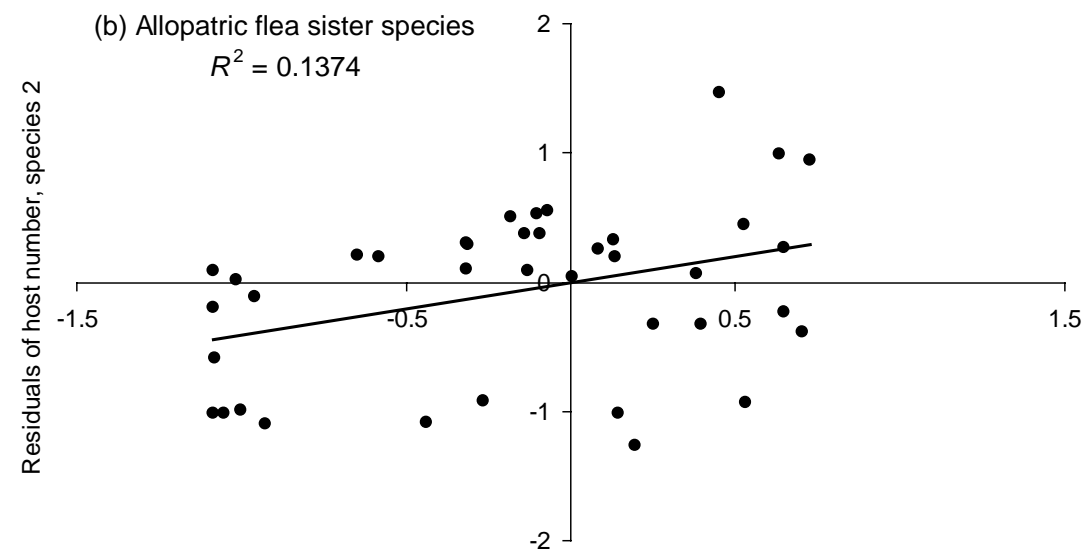

Residuals of host number, species 1

(c) Sympatric flea sister species 1.5

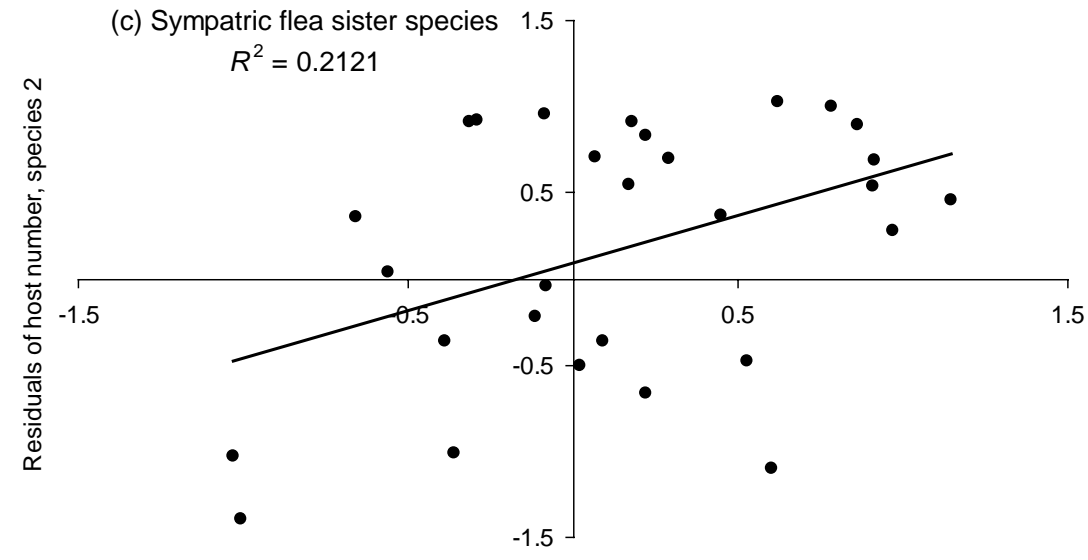

Residuals of host number, species 1

Fig. 1. Plots of host specificity measured as number of host species used by pairs of flea sister species for (a) the whole dataset, (b) only allopatric species, and (c) only sympatric species.

other pattern observed here, that conservatism of host specificity applies to trematodes but not to cestodes or nematodes, also remains unclear. Conservatism of host specificity is not affected by mean host specificity (Table 1) but may be affected by sample size. However, the statistical test is independent from the number of 


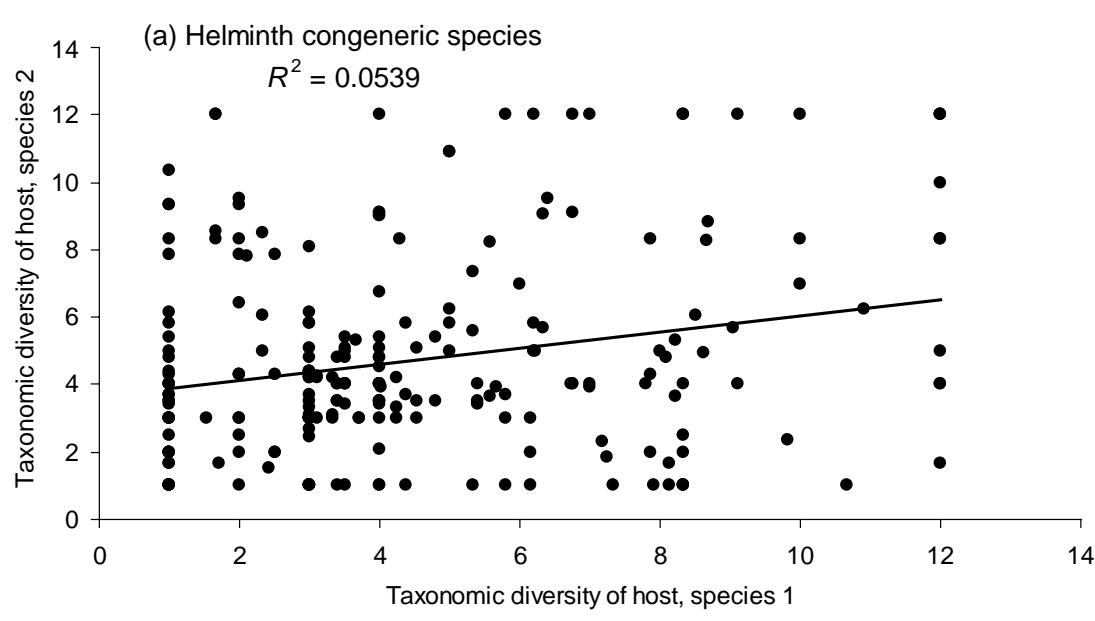

Fig. 2. Plots of host specificity measured as taxonomic distinctness of pairs of (a) helminth congeneric species and (b) of trematode congeneric species.

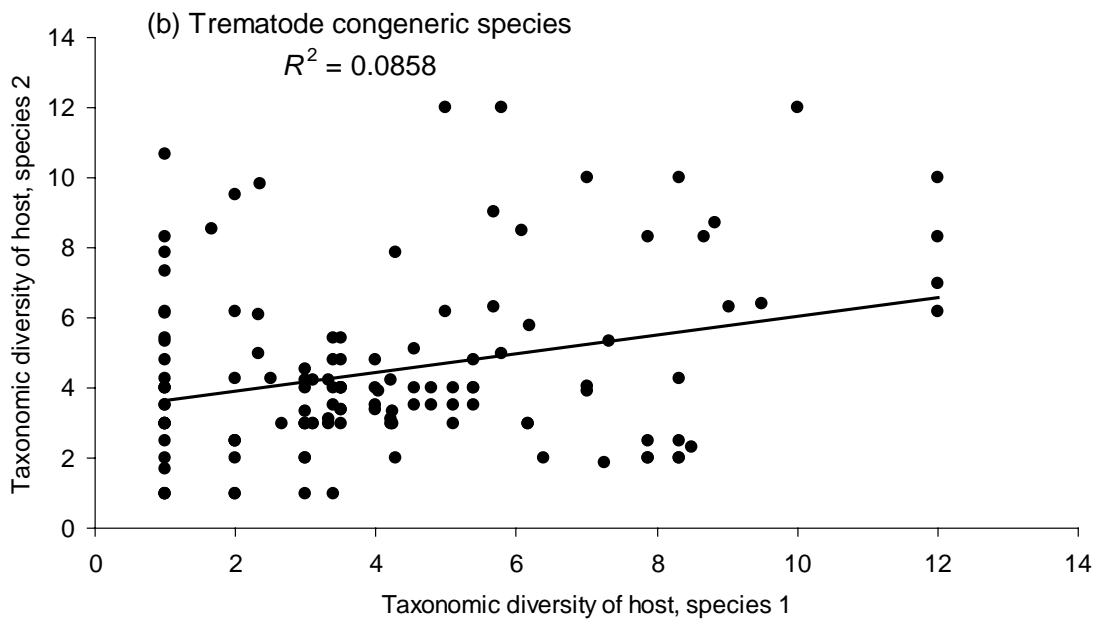

congeneric species or from the number of species pairs included in the analyses as it is based on randomization procedures. We can only hypothesize that the observed differences between helminth groups may come from differences in the number of intermediate hosts (usually 2 for trematodes, and 1 for both cestodes and nematodes). A larger number of intermediate hosts may weaken the conservatism of host specificity by increasing the weight of events related to history and biogeography of host-parasite interactions. The many species-specific parasites, which have been shown to represent up to $30 \%$ of parasites sampled in humans, domesticated animals, and primates (Cleaveland et al. 2001, Pedersen et al. 2005) are not included in host taxonomic diversity as the index needs at least 2 species. Thus our conclusions on the relationships between host specificity (in terms of host taxonomic diversity) of our pairs of species are restricted to parasites infesting more than one host.

In clear contrast to highly conserved traits such as body mass (Desdevises et al. 2003), the relation between the host specificities of closely related parasite species leaves much of the variation unexplained. This renders impossible any prediction of the host specificity of one species based on the specificity of a closely related species (Fig. 1 and 2); we can only state that 2 closely related parasite species are more likely to have similar host specificity than expected by chance.

The patterns reported here for conservatism of host specificity seem very close to the patterns observed for the heritability of the geographic range size in free-living animals, which currently is a topic of intense discussion (Webb and Gaston 2003). Even if the conservatism of external attributes such as host specificity or geographic range size is unambiguously present (most likely due to the conservatism of morphological or biological traits), we suspect that this feature is secondary in its influence on observed patterns, as suggested by Stireman and Singer (2003). Environmental factors, history, and metapopulation dynamics are the main drivers of host specificity and their explanatory power, if any, remains to be tested. 
Acknowledgements - We wish to thank the subject matter editor D. A. Kelt for improving our manuscript with useful comments. This is publication no. 197 of the Ramon Science Center and no. 505 of the Mitrani Dept of Desert Ecology. Experiments comply with the current laws of the country in which they were performed.

\section{References}

Bohning-Gaese, K. and Oberrath, R. 2003. Macroecology of habitat choice in long-distance migratory birds. - Oecologia 137: 296-303.

Brandle, M. et al. 2002. Dietary niche breadth for central European birds: correlations with species-specific traits. - Evol. Ecol. Res. 4: 643-657.

Brown, J. H. 1995. Macroecology. - Univ. of Chicago Press.

Bush, A. O. and Kennedy, C. R. 1994. Host fragmentation and helminth parasites: hedging your bets against extinction. - Int. J. Parasitol. 24: 1333-1343.

Cleaveland, S. et al. 2001. Disease of humans and their domestic mammals: pathogen characteristics, host range and the risk of emergence. - Phil. Trans. R. Soc. B 356: 991-999.

de Meeûs, T. et al. 1998. Santa Rosalia revisited: or why are there so many kinds of parasites in 'The garden of Earthly Delights'? - Parasitol. Today 14: 10-13.

Desdevises, Y. et al. 2003. Quantifying phylogenetically structured environmental variation. - Evolution 57: 2647-2652.

Gaston, K. J. and Blackburn, T. M. 2000. Pattern and process in macroecology. - Blackwell.

Holt, R. D. et al. 2003. Parasite establishment in host communities. - Ecol. Lett. 6: 837-842.

Hopkins, G. H. E. and Rothschild, M. 1953. An illustrated catalogue of the Rothschild collection of fleas Siphonaptera. British Museum Natural History, Vol. I. Tungidae and Pulicidae. - The Trustees of the British Museum, London.

Hopkins, G. H. E. and Rothschild, M. 1956. An illustrated catalogue of the Rothschild collection of fleas Siphonaptera. British Museum Natural History, Vol. II. Coptopsyllidae, Vermipsillidae, Stephanocircidae, Ischnopsyllidae, Hypsophthalmidae and Xiphiopsyllidae. - The Trustees of the British Museum, London.

Hopkins, G. H. E. and Rothschild, M. 1962. An illustrated catalogue of the Rothschild collection of fleas Siphonaptera. British Museum Natural History, Vol. III. Hystrichopsyllidae. - The Trustees of the British Museum, London.

Hopkins, G. H. E. and Rothschild, M. 1966. An illustrated catalogue of the Rothschild collection of fleas Siphonaptera. British Museum Natural History, Vol. IV. Hystrichopsyllidae. - The Trustees of the British Museum, London.

Hopkins, G. H. E. and Rothschild, M. 1971. An illustrated catalogue of the Rothschild collection of fleas Siphonaptera. British Museum Natural History, Vol. V. Leptopsyllidae and Ancistropsyllidae. - The Trustees of the British Museum, London.

Koh, L. P. et al. 2004. Species coextinctions and the biodiversity crisis. - Science 305: 1632-1634.

Krasnov, B. R. et al. 2004a. Ectoparasitic "jacks-of-all-trades": relationship between abundance and host specificity in fleas Siphonaptera parasitic on small mammals. - Am. Nat. 164: 506-516.

Krasnov, B. R. et al. 2004b. Geographical variation in host specificity of fleas (Siphonaptera) parasitic on small mammals: the influence of phylogeny and local environmental conditions. - Ecography 27: 787-797.
Krasnov, B. R. et al. 2005. Host specificity and geographic range in haematophagous ectoparasites. - Oikos 108: 449456.

McCoy, K. D. 2003. Sympatric speciation in parasites: what is sympatry? - Trends Parasitol. 19: 400-404.

Manly, B. F. J. 1998. Randomization, Bootstrap and Monte Carlo methods in biology, 2nd ed. - Chapman and Hall.

Morand, S. and Guégan, J. F. 2000. Distribution and abundance of parasite nematodes: ecological specialisation, phylogenetic constraint or simply epidemiology? - Oikos 88: $563-$ 573.

Pedersen, A. B. et al. 2005. Patterns of host specificity and transmission among parasites of wild primates. - Int. J. Parasitol. 35: 647-657.

Peterson, A. T. et al. 1999. Conservatism of ecological niches in evolutionary time. - Science 285: 1265-1267.

Poulin, R. 1998. Evolutionary ecology of parasites: from individuals to communities. - Chapman and Hall.

Poulin, R. and Mouillot, D. 2003. Parasite specialization from a phylogenetic perspective: a new index of host specificity. - Parasitology 126: 473-480.

Poulin, R. and Mouillot, D. 2004. The relationship between specialization and local abundance: the case of helminth parasites of birds. - Oecologia 140: $372-378$.

Rohde, K. 1979. A critical evaluation of intrinsic and extrinsic factors responsible for niche restriction in parasites. - Am. Nat. 114: 648-671.

Sasal, P. et al. 1998. Host-specialization and species diversity in fish parasites: phylogenetic conservatism? - Ecography 21: 639-643.

Sibley, C. G. and Monroe, B. L. Jr 1990. Distribution and taxonomy of birds of the world. - Yale Univ. Press.

Sibley, C. G. and Ahlquist, J. E. 1991. Phylogeny and classification of birds: a study in molecular evolution. - Yale Univ. Press.

Smit, F. G. A. M. 1987. An illustrated catalogue of the Rothschild collection of fleas Siphonaptera. British Museum Natural History, Vol. V. Malacopsylloidea Malacopsyllidae and Rhopalopsyllidae. - Oxford Univ. Press and The British Museum Natural History.

Stireman, J. O. and Singer, M. S. 2003. What determines host range in parasitoids? An analysis of a tachinid parasitoid community. - Oecologia 135: 629-638.

Théron, A. and Combes, C. 1995. Asynchrony of infection timing, habitat preference, and sympatric speciation of schistosome parasites. - Evolution 49: 372-375.

Traub, R. et al. 1983. The Ceratophyllidae: key to the genera and host relationships. - Cambridge Univ. Press.

Vaidova, S. M. 1978. Helminths of the birds of Azerbaidzhan. - Akademy Nauk Azerbaidzhan SSR, Elm, Baku, in Russian.

Via, S. 2001. Sympatric speciation in animals: the ugly duckling grows up. - Trends Ecol. Evol. 16: 381-390.

Vershinina, T. A. et al. 1967. Fleas of small mammals in the Barguzin depression and their landscape distribution. Proc. of the Irkutsk State Scientific Anti-Plague Inst. of Siberia and Far East 27: 265-278, in Russian.

Webb, T. J. and Gaston, K. J. 2003. On the heritability of geographic range sizes. - Am. Nat. 161: 553-566.

Wilson, D. E. and Reeder, D. M. 1993. Mammal species of the world: a taxonomic and geographic reference. - Smithsonian Inst. Press.

Subject Editor: Douglas Kelt 